Musées, Patrimoine et Culture scientifiques et techniques

$124 \mid 2009$

juillet - août 2009

\title{
Pratiques et enjeux du PSC au sein d'une conservation départementale
}

\section{Chrystèle Burgard}

\section{OpenEdition \\ Journals}

Édition électronique

URL : http://journals.openedition.org/ocim/305

DOI : 10.4000/ocim.305

ISSN : 2108-646X

Éditeur

OCIM

Édition imprimée

Date de publication : 1 juillet 2009

Pagination : $37-39$

ISSN : 0994-1908

Référence électronique

Chrystèle Burgard, « Pratiques et enjeux du PSC au sein d'une conservation départementale », La Lettre de I'OCIM [En ligne], 124 | 2009, mis en ligne le 01 juillet 2011, consulté le 19 avril 2019. URL: http://journals.openedition.org/ocim/305; DOI : 10.4000/ocim.305 


\section{Pratiques et enjeux du PSC au sein $d$ 'une conservation départementale}

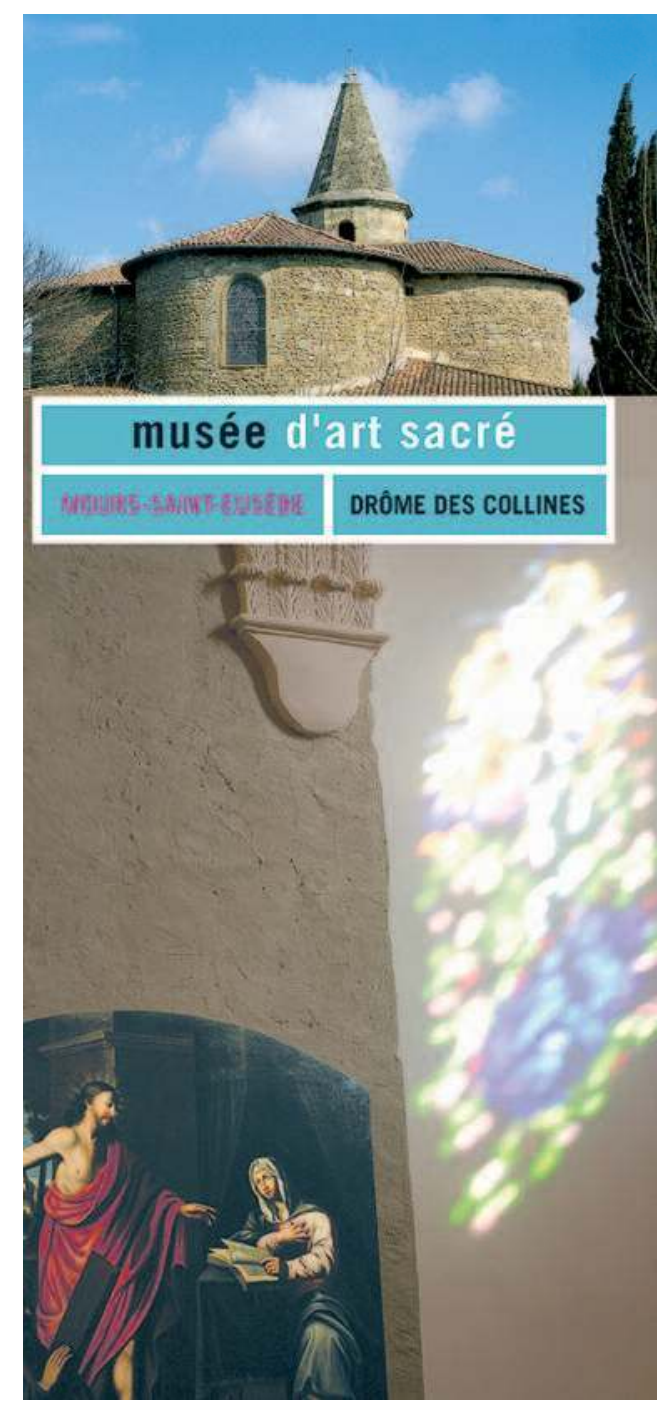

Dépliant de présentation du musée d'Art sacré de Mours-Saint-Eusèbe (c) Conservation départementale des musées de la Drôme

\footnotetext{
* Chrystèle Burgard est conservateur en chef du Patrimoine, responsable de la conservation départementale des musées de la Drôme
}

\section{Chrystèle Burgard *}

À partir de l'expérience de la conservation départementale des musées de la Drôme, I'auteur aborde les différentes questions qui se posent à propos d'un PSC : quelles sont les fonctions du PSC d'un Musée de France situé dans une petite commune ou isolé dans une zone naturelle ? Qui élabore et écrit un PSC ? Quel rôle joue le conservateur?

Ces questions sont soulevées dans le contexte particulier d'une conservation départementale ${ }^{(1)}$ qui remplit des missions d'étude et d'inventaire, de conservation et de restauration, de valorisation et de diffusion des patrimoines d'un département. Dans ce cadre, le conservateur départemental assure la responsabilité scientifique de musées de France qui ne possèdent pas de personnel scientifique comme celui de la Préhistoire du Vercors ou celui d'Art sacré de Mours-SaintEusèbe, la gestion directe de certains musées comme le château-musée de Grignan ou le musée de la Résistance de Vassieux-en-Vercors, enfin il accompagne certains musées appartenant à des collectivités territoriales. Aussi a t-il la charge de plusieurs musées qui seront pour certains rénovés, construits ou simplement réaménagés et il est amené à conduire plusieurs PSC sur des sujets aussi différents que la Préhistoire, l'Art sacré, l'Architecture et les Arts décoratifs ou encore la Résistance, dans des contextes spécifiques où acteurs et attentes sont chaque fois différents.

Il est certain que le conservateur départemental ne joue pas le même rôle qu'un conservateur responsable d'un seul musée, d'une équipe, d'une collection, avec une tutelle unique et qu'il envisage le PSC d'un musée même musée de France avec une stratégie particulière. 
Aborder un PSC de musée situé dans la Drôme, c'est tout d'abord se positionner dans un territoire, un contexte géographique et politique précis et s'inscrire dans une approche départementale à travers le schéma des musées et des maisons thématiques ou le schéma archéologique. Ceux-ci proposent un état des lieux et des orientations, notamment autour de la mise en réseau des sites (supports d'information et de sensibilisation tels dépliants de communication, guide des musées, guide de collections...; logiciel d'informatisation commun...) et de la fédération de certains musées conventionnés avec le Département autour de réserves départementales afin de ne pas multiplier les équipements et le personnel.

Le PSC devient alors un outil pour définir la place et le rôle du musée dans ces schémas (tête de réseau d'une thématique, complémentarité...). Il énonce surtout les enjeux du musée qui dépassent aujourd'hui la seule question de la conservation et de la présentation des collections. Ces enjeux sont multiples et doivent être posés le plus largement possible afin qu'un musée ne soit ni le rêve d'un conservateur solitaire qui y met toutes ses envies et ses frustrations perpétuant alors l'image du conservateur au dessus de toutes contingences - ni la "folie architecturale » d'un homme politique ambitieux qui y voit une manière de marquer son mandat électoral. Un musée est bien un équipement culturel, patrimonial, touristique pour des publics mais il répond également à des

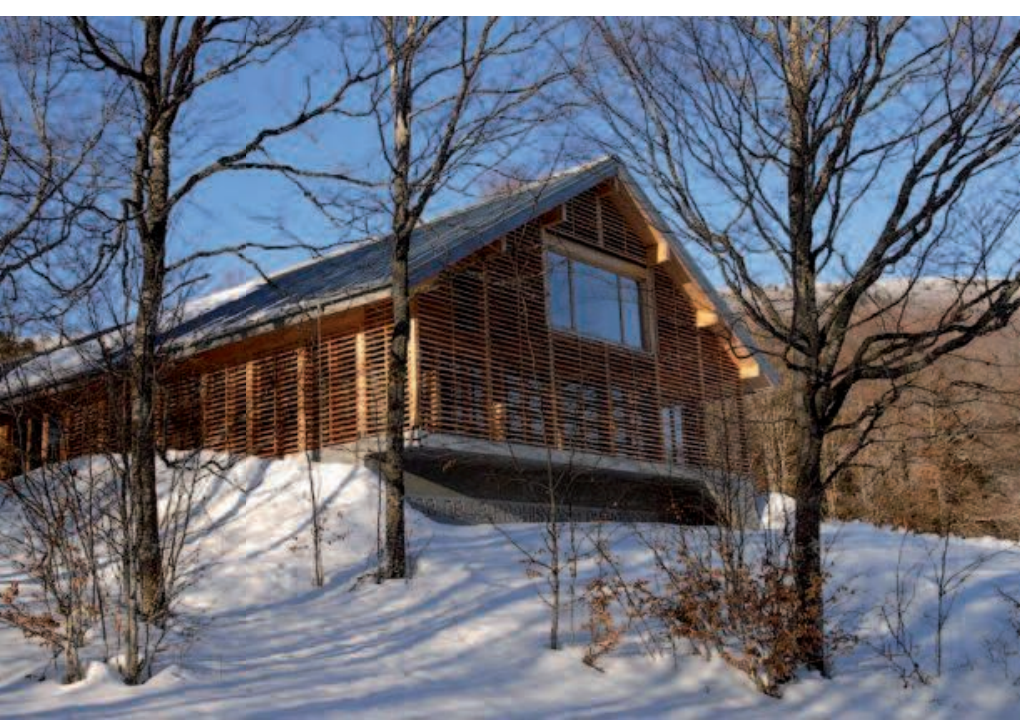

Le musée de la Préhistoire du Vercors à Vassieux-en-Vercors (c) E. Georges enjeux architecturaux, urbains, économiques... Si un PSC doit être un document partagé par différents acteurs, il ne doit pas être pour autant consensuel mais bien un support à une réflexion et un acte d'engagement ; aussi sert-il à confronter des positions, à établir des perspectives, à déterminer des choix sur des questions aussi bien scientifiques que politiques. Il n'est donc pas souhaitable que le conservateur départemental se positionne comme scientifique spécialiste d'un domaine ; il doit plutôt être du côté du coordonnateur voire du médiateur, capable de rédiger un projet réalisable sans être dégagé de l'opérationnel comme le préconise la Direction des Musées de France, capable de s'appuyer sur des points de vue tout en proposant une direction affirmée.

\section{Un enjeu scientifique et territorial : le musée de la Préhistoire du Vercors}

Construit autour d'un atelier de taille de silex abandonné il y 4500 ans, le musée de la Préhistoire du Vercors à Vassieux-en-Vercors est un "Musée de France » et un site classé Monument historique, géré par le Parc naturel régional du Vercors et sous la responsabilité scientifique de la Conservation du patrimoine de la Drôme.

L'enjeu du PSC était de concevoir un projet répondant aux attentes du Parc naturel dont la zone géographique couvre partiellement deux départements, du Conseil général de la Drôme, de la Région Rhône-Alpes, d'une Direction régionale des Affaires culturelles Rhône-Alpes où trois services étaient impliqués : archéologie, musées, monuments historiques. Il était de définir un projet scientifique avec des préhistoriens - spécialistes de périodes, inventeurs du site - attachés chacun à des problématiques précises, impliqués chacun de manière plus ou moins affective. Il était d'aborder la question cruciale du statut du mobilier archéologique, peu claire entre les musées et les archéologues. Il était de requalifier l'architecture de ce musée en rénovant le bâtiment existant bâti autour du site de taille et en créant une extension contemporaine en adéquation avec le contenu du site, des collections modestes, un environnement naturel fort, et aussi de donner de véritables espaces pour développer l'accueil de groupes scolaires dans le cadre d'ateliers ou de démonstration de taille. Dans ce contexte, le rôle du conservateur était avant tout de coordonner les acteurs aussi bien scientifiques que politiques, avec la forte implication de la direction du parc. 
De ces contraintes et attentes, sont nées des orientations scientifiques et culturelles centrées sur les relations de l'homme et de la montagne pendant la Préhistoire dans le Vercors, sur la circulation des hommes et de leur savoir-faire (chasse, taille de silex...), à travers trente années de recherches archéologiques conduites sur ce territoire. Après deux années d'élaboration de PSC, une année de programmation muséographique et de montage financier, un an et demi de travaux, le musée a ouvert ses portes au public l'été 2008.

\section{Un enjeu culturel/cultuel : le musée d'Art sacré de Mours-Saint-Eusèbe}

La collection de plus de 28000 objets et œuvres d'Art sacré, propriété de l'association diocésaine, attend depuis une trentaine années d'être présentée et conservée dans un véritable musée, malgré plusieurs PSC et projets. Ce «Musée de France » sans espace spécifique en dehors de l'église est géré par l'association des Amis du musée et placé sous la responsabilité scientifique de la Conservation du patrimoine. L'enjeu du PSC était cette fois-ci de définir un contenu qui ne soit pas destiné au seul milieu catholique, de concevoir un musée avec une vocation culturelle et non cultuelle avec des acteurs croyants et d'autres athées. Sa définition s'est établie de manière collective en s'appuyant sur des travaux d'étudiants, d'enseignants, de chercheurs en particulier en ethnologie pour dépasser l'approche esthétique d'un musée d'Art sacré, en affirmant une démarche anthropologique, en positionnant le musée comme lieu d'analyse et de questionnements pour tous les publics et comme équipement culturel laïque financé avec des fonds publics.

Il est proposé une approche pluridisciplinaire de l'expérience religieuse dans la Drôme afin de comprendre comment la religion a structuré l'espace et façonné l'homme, notamment en évoquant les pratiques sociales et la dévotion populaire dans le département. L'intention du projet est double, d'une part redonner à l'église sa fonction cultuelle, comme espace de l'expérience de la foi, d'autre part construire un musée dissocié de l'église, comme espace

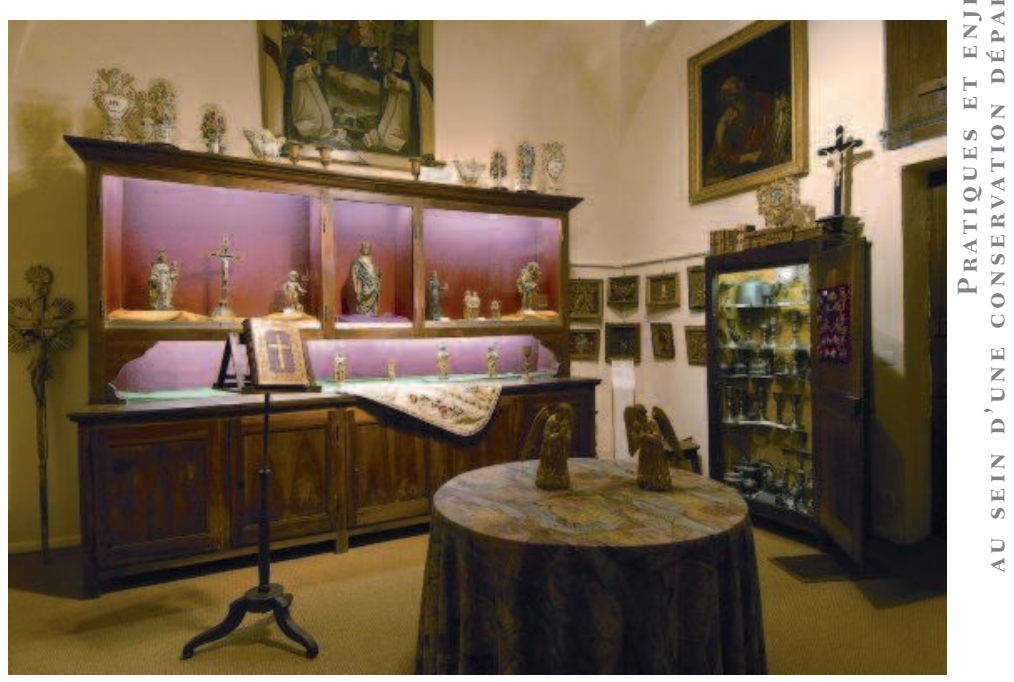

Intérieur du musée d'Art sacré de Mours-Saint-Eusèbe (c) E. Georges

de découverte culturelle et de plaisir. Après deux années pour réaliser le PSC, une année pour l'étude de programmation, le projet architectural pourrait commencer prochainement.

À travers ces deux exemples, il m'importait de témoigner de la nécessité d'élaborer un projet pour chaque musée, monument historique ou maison thématique, qu'il soit sous forme " calibrée » de PSC pour les Musées de France, sous forme de schéma directeur pour les uns ou de projet de musée pour les autres; de témoigner de l'intérêt de cette phase constructive qui se révèle aussi enrichissante que celle de la gestion et du fonctionnement d'un musée : le temps du projet où convergent réflexions et perspectives.

\section{Note}

(1) Pour réaliser ces missions, l'équipe de la conservation départementale des musées de la Drôme est composée de 8 personnes dont C. Marande, chargée des musées. 